\title{
Nonuniform Dependence on Initial Data of a Periodic Camassa-Holm System
}

\author{
Xiaohuan Wang \\ College of Mathematics and Information Science, Henan University, Kaifeng 475001, China \\ Correspondence should be addressed to Xiaohuan Wang; xiaohuanw@126.com
}

Received 22 November 2013; Accepted 21 January 2014; Published 4 March 2014

Academic Editor: Ziemowit Popowicz

Copyright ( 2014 Xiaohuan Wang. This is an open access article distributed under the Creative Commons Attribution License, which permits unrestricted use, distribution, and reproduction in any medium, provided the original work is properly cited.

This paper is concerned with some properties of a periodic two-component Camassa-Holm system. By constructing two sequences of solutions of the two-component Camassa-Holm system, we prove that the solution map of the Cauchy problem of the twocomponent Camassa-Holm system is not uniformly continuous in $H^{s}(\mathbb{S}), s>5 / 2$.

\section{Introduction}

In this paper, we consider the Cauchy problem of the following two-component periodic Camassa-Holm system:

$$
\begin{gathered}
m_{t}+u m_{x}+2 m u_{x}=-\rho \rho_{x}, \quad t>0, x \in \mathbb{R}, \\
\rho_{t}+(\rho u)_{x}=0, \quad t>0, x \in \mathbb{R}, \\
u(0, x)=u_{0}(x), \quad \rho(0, x)=\rho_{0}(x), \quad x \in \mathbb{R}, \\
u(t, x+1)=u(t, x), \quad \rho(t, x+1)=\rho(t, x), \\
t \geq 0, x \in \mathbb{R},
\end{gathered}
$$

where $m=u-u_{x x}$. The Camassa-Holm equation can be obtained via the obvious reduction $\rho \equiv 0$.

The Camassa-Holm $(\mathrm{CH})$ equation has been extended to a two-component integrable system $(\mathrm{CH} 2)$ by combining its integrability property with compressibility, or free-surface elevation dynamics in its shallow-water interpretation $[1,2]$; that is,

$$
\begin{gathered}
m_{t}+u m_{x}+2 m u_{x}+\sigma \rho \rho_{x}=0, \quad t>0, x \in \mathbb{R}, \\
\rho_{t}+(\rho u)_{x}=0, \quad t>0, \quad x \in \mathbb{R},
\end{gathered}
$$

where $m=u-u_{x x}$ and $\sigma= \pm 1$. Local well-posedness of system (2) with $\sigma=1$ was obtained by $[1,3]$. The precise blowup scenarios and blow-up phenomena of strong solution for system (2) was established by [1,3-6]. Just recently, Gui and Liu [7] studied system (1) with $\sigma=1$ in Besov space and they obtained the local well-posedness. In this paper, we consider the Cauchy problem of system (1) and study some properties of it.

If $\rho \equiv 0$, then system (2) becomes the well-known Camassa-Holm equation [8]. In the past decade, the Camassa-Holm equation has attracted much attention because of its integrability and the existence of multipeakon solution; see [4, 8-22] for the details. The Cauchy problem and initial boundary value problem of the Camassa-Holm equation have been studied extensively $[10,23]$. It has been shown that the Camassa-Holm equation is locally wellposedness [10] for initial data $u_{0} \in H^{s}(\mathbb{S}), s>3 / 2$. Moreover, it has global strong solutions $[10,18]$ and finite time blow-up solutions [10]. On the other hand, it has global weak solution in $H^{1}(\mathbb{S})[8,9,13,19]$. The advantage of the Camassa-Holm equation in comparison with the KdV equation lies in the fact that the Camassa-Holm equation has peaked solutions and models wave breaking (i.e., the solution remains bounded while its slope becomes unbounded in finite time $[8,10,24])$.

Recently, some properties of solutions to the CamassaHolm equation have been studied by many authors. Himonas et al. [15] studied the persistence properties and unique continuation of solutions of the Camassa-Holm equation. They showed that a strong solution of the Camassa-Holm equation, initially decaying exponentially together with its spatial derivative, must be identically equal to zero if it also 
decays exponentially at a later time; see [11, 22] for the same properties of solutions to other shallow water equations. Just recently, Himonas and Kenig [16] and Himonas et al. $[14,17]$ considered the nonuniform dependence on initial data for the Camassa-Holm equation on the line and on the circle, respectively. $\mathrm{Lv}$ et al. [25] obtained the nonuniform dependence on initial data for $\mu-b$ equation. Lv and Wang [26] considered the system (1) with $\rho=$ $\gamma-\gamma_{x x}$ and obtained the nonuniform dependence on initial data.

In this paper, we will consider the nonuniform dependence on initial data to system (1). We remark that there is significant difference between system (1) and system (1) with $\rho=\gamma-\gamma_{x x}$. It is easy to see that when $\rho=\gamma-\gamma_{x x}$, there are some similar properties between the two equations in system (1). Thus the proof of nonuniform dependence on initial data to system (1) with $\rho=\gamma-\gamma_{x x}$ is similar to the single equation, for example, Camassa-Holm equation. But in system (1), $\rho$ and $u$ have different properties; see Theorem 1. This needs constructing different asymptotic solution; see Section 3 .

This paper is organized as follows. In Section 2, we recall the well-posedness result of $\mathrm{Hu}$ and Yin [27] and use it to prove the basic energy estimate from which we derive a lower bound for the lifespan of the solution as well as an estimate of the $H^{s}(\mathbb{S}) \times H^{s-1}(\mathbb{S})$ norm of the solution $(u(t, x), \rho(t, x))$ in terms of $H^{s}(\mathbb{S}) \times H^{s-1}(\mathbb{S})$ norm of the initial data $\left(u_{0}, \rho_{0}\right)$. In Section 3, we construct approximate solutions, compute the error, and estimate the $H^{1}$-norm of this error. In Section 4, we estimate the difference between approximate and actual solutions, where the exact solution is a solution to system (1) with initial data given by the approximate solutions evaluated at time zero. The nonuniform dependence on initial data for system (1) is established in Section 5 by constructing two sequences of solutions to system (1) in a bounded subset of the Sobolev space $H^{S}(\mathbb{S})$, whose distance at the initial time is converging to zero while at any later time it is bounded below by a positive constant. During preparing our paper, we find another paper [28] where the same problem has been considered, but our method is different from theirs.

Notation. In the following, we denote by $*$ the spatial convolution. Given a Banach space $Z$, we denote its norm by $\|\cdot\|_{Z}$. Since all space of functions are over $\mathbb{S}$, for simplicity, we drop $\mathbb{S}$ in our notations of function spaces if there is no ambiguity. Let $[A, B]=A B-B A$ denote the commutator of linear operator $A$ and $B$; see $[29,30]$ for the details. Set $\|z\|_{H^{s} \times H^{s-1}}^{2}=\|u\|_{H^{s}}^{2}+\|\rho\|_{H^{s-1}}^{2}$, where $z=(u, \rho)$.

\section{Local Well-Posedness}

In this section we first recall the known results of $\mathrm{Hu}$ and Yin [27] and give a new estimate of the solution to (1).

Let $\Lambda=\left(1-\partial_{x}^{2}\right)^{1 / 2}$. Then the operator $\Lambda^{-2}$ acting on $L^{2}(\mathbb{S})$ can be expressed by its associated Green's function
$G(x)=\cosh (x-[x]-(1 / 2)) / 2 \sinh (1 / 2)$, where $[x]$ stands for the integer part of $x$, as

$$
\begin{aligned}
& \Lambda^{-2} f(x)=(G * f)(x) \\
&=\frac{1}{2} \int_{\mathbb{S}} \frac{\cosh (x-y-[x-y]-(1 / 2))}{\sinh (1 / 2)} f(y) d y, \\
& f \in L^{2}(\mathbb{S}) .
\end{aligned}
$$

Hence (1) is equivalent to the following system:

$$
\begin{gathered}
u_{t}+u u_{x}=-\partial_{x} \Lambda^{-2}\left(u^{2}+\frac{1}{2} u_{x}^{2}+\frac{1}{2} \rho^{2}\right), \\
t>0, x \in \mathbb{R}, \\
\rho_{t}+u \rho_{x}=-u_{x} \rho, \quad t>0, x \in \mathbb{R}, \\
u(0, x)=u_{0}(x), \quad \rho(0, x)=\rho_{0}(x), x \in \mathbb{R}, \\
u(t, x+1)=u(t, x), \quad \rho(t, x+1)=\rho(t, x), \\
t \geq 0, x \in \mathbb{R} .
\end{gathered}
$$

In the rest of this paper, we will consider the following system:

$$
\begin{gathered}
u_{t}+u u_{x}=-\partial_{x} \Lambda^{-2}\left(u^{2}+\frac{1}{2} u_{x}^{2}+\frac{1}{2} \rho^{2}\right), \\
t>0, x \in \mathbb{S}, \\
\rho_{t}+u \rho_{x}=-u_{x} \rho, \quad t>0, x \in \mathbb{S}, \\
u(0, x)=u_{0}(x), \quad \rho(0, x)=\rho_{0}(x), \quad x \in \mathbb{S} .
\end{gathered}
$$

The following result is obtained by $\mathrm{Hu}$ and Yin [27].

Theorem 1 (see [27]). Given $z_{0}=\left(u_{0}, \rho_{0}\right) \in H^{s} \times H^{s-1}$, $s \geq 2$. Then there exists a maximal existence time $T=$ $T\left(\left\|z_{0}\right\|_{H^{s} \times H^{s-1}}\right)>0$ and a unique solution $z=(u, \rho)$ to system (5) such that

$$
\begin{aligned}
z= & z\left(\cdot, z_{0}\right) \\
& \quad \in C\left([0, T) ; H^{s} \times H^{s-1}\right) \cap C^{1}\left([0, T) ; H^{s-1} \times H^{s-2}\right) .
\end{aligned}
$$

Moreover, the solution depends continuously on the initial data; that is, the mapping

$$
\begin{aligned}
z_{0} \longrightarrow & z\left(\cdot, z_{0}\right): H^{s} \times H^{s-1} \\
\longrightarrow & C\left([0, T) ; H^{s} \times H^{s-1}\right) \\
& \cap C^{1}\left([0, T) ; H^{s-1} \times H^{s-2}\right)
\end{aligned}
$$

is continuous.

Next, we will give an explicit estimate for the maximal existence time $T$. Also, we will show that at any time $t$ in 
the time interval $\left[0, T_{0}\right]$ the $H^{s}$-norm of the solution $z(t, x)$ is dominated by the $H^{s}$-norm of the initial data $z_{0}(x)$. In order to do this, we need the following lemmas.

Lemma 2 (see [29]). If $r>0$, then $H^{r} \cap L^{\infty}$ is an algebra. Moreover,

$$
\|f g\|_{H^{r}} \leq C\left(\|f\|_{\infty}\|g\|_{H^{r}}+\|f\|_{H^{r}}\|g\|_{\infty}\right),
$$

where $C$ is a positive constant depending only on $r$.

Lemma 3 (see [29]). If $r>0$, then

$$
\left\|\left[\Lambda^{r}, f\right] g\right\|_{2} \leq C\left(\left\|f_{x}\right\|_{\infty}\left\|\Lambda^{r-1} g\right\|_{2}+\left\|\Lambda^{r} f\right\|_{2}\|g\|_{\infty}\right),
$$

where $C$ is a positive constant depending only on $r$.

Theorem 4. Let $s>5 / 2$. If $z=(u, \rho)$ is a solution of system (5) with initial data $z_{0}$ described in Theorem 1 , then the maximal existence time $T$ satisfies

$$
T \geq T_{0}:=\frac{1}{2 C_{s}\left\|z_{0}\right\|_{H^{s} \times H^{s-1}}},
$$

where $C_{s}$ is a constant depending only on s. Also, we have

$$
\|z(t)\|_{H^{s} \times H^{s-1}} \leq 2\left\|z_{0}\right\|_{H^{s} \times H^{s-1}}, \quad 0 \leq t \leq T_{0} .
$$

Proof. The derivation of the lower bound for the maximal existence time (10) and the solution size estimate (11) is based on the following differential inequality for the solution $z$ :

$$
\frac{1}{2} \frac{d}{d t}\|z(t)\|_{H^{s} \times H^{s-1}}^{2} \leq C_{s}\|z(t)\|_{H^{s} \times H^{s-1}}^{3}, \quad 0 \leq t<T .
$$

Suppose that (12) holds. Then, integrating (12) from 0 to $t$, we have

$$
\|z(t)\|_{H^{s} \times H^{s-1}} \leq \frac{\left\|z_{0}\right\|_{H^{s} \times H^{s-1}}}{1-C_{s}\left\|z_{0}\right\|_{H^{s} \times H^{s-1}} t} .
$$

It follows from the above inequality that $\|z(t)\|_{H^{s} \times H^{s-1}}$ is finite if $C_{s}\left\|z_{0}\right\|_{H^{s} \times H^{s-1}} t<1$. Let $T_{0}=1 / 2 C_{s}\left\|z_{0}\right\|_{H^{s} \times H^{s-1}}$, then, for $0 \leq t \leq T_{0}$, we have

$$
\|z(t)\|_{H^{s} \times H^{s-1}} \leq \frac{\left\|z_{0}\right\|_{H^{s} \times H^{s-1}}}{1-C_{s}\left\|z_{0}\right\|_{H^{s} \times H^{s-1}} T_{0}}=2\left\|z_{0}\right\|_{H^{s} \times H^{s-1}} .
$$

Now we prove inequality (12). Note that the products $u u_{x}$ and $u \rho_{x}$ are only in $H^{s-1}$ if $u, \rho \in H^{s}$. To deal with this problem, we will consider the following modified system:

$$
\begin{gathered}
\left(J_{\varepsilon} u\right)_{t}+J_{\varepsilon}\left(u u_{x}\right) \\
=-\partial_{x} \Lambda^{-2}\left(J_{\varepsilon} u^{2}+\frac{1}{2} J_{\varepsilon} u_{x}^{2}+\frac{1}{2} J_{\varepsilon} \rho^{2}\right), \\
t>0, x \in \mathbb{S} \\
\left(J_{\varepsilon} \rho\right)_{t}+J_{\varepsilon}\left(u \rho_{x}\right)=-J_{\varepsilon}\left(u_{x} \rho\right), \quad t>0, x \in \mathbb{S},
\end{gathered}
$$

where for each $\varepsilon \in(0,1]$ the operator $J_{\varepsilon}$ is the Friedrichs mollifier defined by

$$
J_{\varepsilon} f(x)=J_{\varepsilon}(f)(x)=j_{\varepsilon} * f .
$$

Here $j_{\varepsilon}(x)=(1 / \varepsilon) j(x / \varepsilon)$, and $j(x)$ is a $C^{\infty}$ function supported in the interval $[-1,1]$ such that $j(x) \geq 0, \int_{\mathbb{S}} j(x) d x=$ 1. Applying the operator $\Lambda^{s}$ and $\Lambda^{s-1}$ to the first and second equations of (15), respectively, then multiplying the resulting equations by $\Lambda^{s} J_{\varepsilon} u$ and $\Lambda^{s-1} J_{\varepsilon} \rho$, respectively, and integrating them with respect to $x \in \mathbb{S}$, we obtain

$$
\begin{aligned}
\frac{1}{2} \frac{d}{d t}\left\|J_{\varepsilon} u\right\|_{H^{s}}^{2}= & -\int_{\mathbb{S}} \Lambda^{s} J_{\varepsilon}\left(u u_{x}\right) \Lambda^{s} J_{\varepsilon} u d x \\
& -\int_{\mathbb{S}} \partial_{x} \Lambda^{s-2} \partial_{x} \Lambda^{-2}\left(J_{\varepsilon} u^{2}+\frac{1}{2} J_{\varepsilon} u_{x}^{2}\right. \\
& \left.+\frac{1}{2} J_{\varepsilon} \rho^{2}\right) \Lambda^{s} J_{\varepsilon} u d x
\end{aligned}
$$

$$
\begin{aligned}
\frac{1}{2} \frac{d}{d t}\left\|J_{\varepsilon} \rho\right\|_{H^{s-1}}^{2}= & -\int_{\mathbb{S}} \Lambda^{s-1} J_{\varepsilon}\left(u \rho_{x}\right) \Lambda^{s-1} J_{\varepsilon} \rho d x \\
& -\int_{\mathbb{S}} \Lambda^{s-1} J_{\varepsilon}\left(u_{x} \rho\right) \Lambda^{s-1} J_{\varepsilon} \rho d x
\end{aligned}
$$

We estimate the right-hand sides of (17) and (18), and we will use the fact that $\Lambda^{s}$ and $J_{\varepsilon}$ are commutative and

$$
\left(J_{\varepsilon} f, g\right)_{0}=\left(f, J_{\varepsilon} g\right)_{0}, \quad\left\|J_{\varepsilon} u\right\|_{H^{s}} \leq\|u\|_{H^{s}} .
$$

To estimate the first integrals in the right-hand sides of (17) and (18) we write them as follows:

$$
\begin{aligned}
\int_{\mathbb{S}} \Lambda^{s} J_{\varepsilon}\left(u u_{x}\right) \Lambda^{s} J_{\varepsilon} u d x= & \int_{\mathbb{S}} \Lambda^{s}\left(u u_{x}\right) J_{\varepsilon} \Lambda^{s} J_{\varepsilon} u d x \\
= & \left(\left[\Lambda^{s}, u\right] u_{x}, J_{\varepsilon} \Lambda^{s} J_{\varepsilon} u\right)_{0} \\
& +\left(u \Lambda^{s} u_{x}, J_{\varepsilon} \Lambda^{s} J_{\varepsilon} u\right)_{0}, \\
\int_{\mathbb{S}} \Lambda^{s-1} J_{\varepsilon}\left(u \rho_{x}\right) \Lambda^{s-1} J_{\varepsilon} \rho d x= & \int_{\mathbb{S}} \Lambda^{s-1}\left(u \rho_{x}\right) J_{\varepsilon} \Lambda^{s-1} J_{\varepsilon} \rho d x \\
= & \left(\left[\Lambda^{s-1}, u\right] \rho_{x}, J_{\varepsilon} \Lambda^{s-1} J_{\varepsilon} \rho\right)_{0} \\
& +\left(u \Lambda^{s-1} \rho_{x}, J_{\varepsilon} \Lambda^{s-1} J_{\varepsilon} \rho\right)_{0} .
\end{aligned}
$$

Using Lemma 3 and (19), we can estimate the first part in the right-hand sides of (20)

$$
\begin{aligned}
\left|\left(\left[\Lambda^{s}, u\right] u_{x}, J_{\varepsilon} \Lambda^{s} J_{\varepsilon} u\right)_{0}\right| \leq & \left\|\left[\Lambda^{s}, u\right] u_{x}\right\|_{2}\left\|J_{\varepsilon} \Lambda^{s} J_{\varepsilon} u\right\|_{2} \\
\leq & C_{s}\left\|u_{x}\right\|_{\infty}\|u\|_{H^{s}}^{2}, \\
\left|\left(\left[\Lambda^{s-1}, u\right] \rho_{x}, J_{\varepsilon} \Lambda^{s-1} J_{\varepsilon} \rho\right)_{0}\right| \leq & \left\|u_{x}\right\|_{\infty}\|\rho\|_{H^{s-1}}^{2} \\
& +\left\|\rho_{x}\right\|_{\infty}\|u\|_{H^{s}}\|\rho\|_{H^{s-1}},
\end{aligned}
$$


where we use the fact that $\|u\|_{H^{s}}=\left\|\Lambda^{s} u\right\|_{2}$. Noting that

$$
\left\|\left[J_{\varepsilon}, u\right] f_{x}\right\|_{2} \leq C\left\|u_{x}\right\|_{\infty}\|f\|_{2},
$$

which is obtained by Himonas and Kenig (see [16, Lemma 2]), and integrating by parts, we obtain

$$
\begin{aligned}
\left|\left(u \Lambda^{s} u_{x}, J_{\varepsilon} \Lambda^{s} J_{\varepsilon} u\right)_{0}\right|= & \left|\left(J_{\varepsilon} u \Lambda^{s} u_{x}, \Lambda^{s} J_{\varepsilon} u\right)_{0}\right| \\
= & \mid\left(\left[J_{\varepsilon}, u\right] \partial_{x} \Lambda^{s} u, \Lambda^{s} J_{\varepsilon} u\right)_{0} \\
& +\left(u J_{\varepsilon} \partial_{x} \Lambda^{s} u, \Lambda^{s} J_{\varepsilon} u\right)_{0} \mid \\
\leq & \left\|\left[J_{\varepsilon}, u\right] \partial_{x} \Lambda^{s} u\right\|_{2}\|u\|_{H^{s}} \\
& +\frac{1}{2}\left|\left(u_{x} \Lambda^{s} J_{\varepsilon} u, \Lambda^{s} J_{\varepsilon} u\right)_{0}\right| \\
\leq & C_{s}\left\|u_{x}\right\|_{\infty}\|u\|_{H^{s}}^{2}, \\
\left|\left(u \Lambda^{s-1} \rho_{x}, J_{\varepsilon} \Lambda^{s-1} J_{\varepsilon} \rho\right)_{0}\right|= & \left|\left(\left[J_{\varepsilon}, u\right] \partial_{x} \Lambda^{s-1} \rho, \Lambda^{s-1} J_{\varepsilon} \rho\right)_{0}\right| \\
& +\frac{1}{2}\left|\left(u_{x} \Lambda^{s-1} J_{\varepsilon} \rho, \Lambda^{s-1} J_{\varepsilon} \rho\right)_{0}\right| \\
\leq & C_{s}\left\|u_{x}\right\|_{\infty}\|\rho\|_{H^{s-1}}^{2} .
\end{aligned}
$$

Combining (21)-(24), we have

$$
\begin{aligned}
& \left|\int_{\mathbb{S}} \Lambda^{s} J_{\varepsilon}\left(u u_{x}\right) \Lambda^{s} J_{\varepsilon} u d x\right| \leq C_{s}\left\|u_{x}\right\|_{\infty}\|u\|_{H^{s}}^{2}, \\
& \left|\int_{\mathbb{S}} \Lambda^{s-1} J_{\varepsilon}\left(u \rho_{x}\right) \Lambda^{s-1} J_{\varepsilon} \rho d x\right| \\
& \quad \leq C_{s}\left(\left\|u_{x}\right\|_{\infty}+\left\|\rho_{x}\right\|_{\infty}\right)\left(\|u\|_{H^{s}}^{2}+\|\rho\|_{H^{s-1}}^{2}\right) .
\end{aligned}
$$

For the second integral in the right-hand side of (17), we have

$$
\begin{aligned}
& \left|\int_{\mathbb{S}} \partial_{x} \Lambda^{s-2}\left(J_{\varepsilon} u^{2}+\frac{1}{2} J_{\varepsilon} u_{x}^{2}+\frac{1}{2} J_{\varepsilon} \rho^{2}\right) \Lambda^{s} J_{\varepsilon} u d x\right| \\
& \quad \leq\left\|\partial_{x} \Lambda^{s-2}\left(J_{\varepsilon} u^{2}+\frac{1}{2} J_{\varepsilon} u_{x}^{2}+\frac{1}{2} J_{\varepsilon} \rho^{2}\right)\right\|_{2}\|u\|_{H^{s}} \\
& \quad \leq\left\|J_{\varepsilon} u^{2}+\frac{1}{2} J_{\varepsilon} u_{x}^{2}+\frac{1}{2} J_{\varepsilon} \rho^{2}\right\|_{H^{s-1}}\|u\|_{H^{s}} \\
& \quad \leq C_{s}\left(\|u\|_{\infty}+\|\rho\|_{\infty}+\left\|u_{x}\right\|_{\infty}\right)\left(\|u\|_{H^{s}}^{2}+\|\rho\|_{H^{s-1}}^{2}\right),
\end{aligned}
$$

where we have used Lemma 2 with $r=s-1$. Similarly, for the second and third integrals in the right-hand side of (18), we get

$$
\begin{aligned}
\left|\int_{S} \Lambda^{s-1} J_{\varepsilon}\left(u_{x} \rho\right) \Lambda^{s-1} J_{\varepsilon} \rho d x\right| & \leq\left\|\rho u_{x}\right\|_{H^{s-1}}\|\rho\|_{H^{s-1}} \\
& \leq C_{s}\left\|u_{x}\right\|_{\infty}\|\rho\|_{H^{s-1}}^{2} .
\end{aligned}
$$

Submitting (25), (27), and (26), (28) into (17) and (18), respectively, we obtain

$$
\begin{aligned}
\frac{1}{2} \frac{d}{d t}\left\|J_{\varepsilon} u\right\|_{H^{s}}^{2} \leq & C_{s}\left(\|u\|_{\infty}+\|\rho\|_{\infty}+\left\|u_{x}\right\|_{\infty}\left\|\rho_{x}\right\|_{\infty}\right) \\
& \times\left(\|u\|_{H^{s}}^{2}+\|\rho\|_{H^{s-1}}^{2}\right), \\
\frac{1}{2} \frac{d}{d t}\left\|J_{\varepsilon} \rho\right\|_{H^{s-1}}^{2} \leq & C_{s}\left(\|u\|_{\infty}+\|\rho\|_{\infty}+\left\|u_{x}\right\|_{\infty}+\left\|\rho_{x}\right\|_{\infty}\right) \\
& \times\left(\|u\|_{H^{s}}^{2}+\|\rho\|_{H^{s-1}}^{2}\right) .
\end{aligned}
$$

Consequently,

$$
\begin{aligned}
\frac{1}{2} \frac{d}{d t} & \left(\left\|J_{\varepsilon} u\right\|_{H^{s}}^{2}+\left\|J_{\varepsilon} \rho\right\|_{H^{s-1}}^{2}\right) \\
\leq & C_{s}\left(\|u\|_{\infty}+\|\rho\|_{\infty}+\left\|u_{x}\right\|_{\infty}+\left\|\rho_{x}\right\|_{\infty}\right) \\
& \times\left(\|u\|_{H^{s}}^{2}+\|\rho\|_{H^{s-1}}^{2}\right) .
\end{aligned}
$$

Then, letting $\varepsilon$ go to 0 , we have

$$
\begin{aligned}
\frac{1}{2} \frac{d}{d t} & \left(\|u\|_{H^{s}}^{2}+\|\rho\|_{H^{s-1}}^{2}\right) \\
\leq & C_{s}\left(\|u\|_{\infty}+\|\rho\|_{\infty}+\left\|u_{x}\right\|_{\infty}+\left\|\rho_{x}\right\|_{\infty}\right) \\
& \times\left(\|u\|_{H^{s}}^{2}+\|\rho\|_{H^{s-1}}^{2}\right),
\end{aligned}
$$

or

$$
\begin{aligned}
\frac{1}{2} \frac{d}{d t}\|z(t)\|_{H^{s} \times H^{s-1}}^{2} \leq & C_{s}\left(\|u(t)\|_{C^{1}}+\|\rho\|_{C^{1}}\right) \\
& \times\|z(t)\|_{H^{s} \times H^{s-1}}^{2} .
\end{aligned}
$$

Since $s>5 / 2$, using Sobolev's inequality we have that

$$
\|u(t)\|_{C^{1}} \leq C_{s}\|u(t)\|_{H^{s}}, \quad\|\rho(t)\|_{C^{1}} \leq C_{s}\|\rho(t)\|_{H^{s-1}} .
$$

From (32) we obtain the desired inequality (12). This completes the proof of Theorem 4 .

Recall that $\|z(t)\|_{H^{s} \times H^{s-1}}^{2}=\|u(t)\|_{H^{s}}^{2}+\|\rho(t)\|_{H^{s-1}}^{2}$, where $z(t)=(u(t), \rho(t))$. It follows from Theorem 4 that

$$
\begin{aligned}
\|u(t)\|_{H^{s}},\|\rho(t)\|_{H^{s-1}} & \leq\|z(t)\|_{H^{s} \times H^{s-1}} \\
& \leq 2\left\|z_{0}\right\|_{H^{s} \times H^{s-1}}, \quad 0 \leq t \leq T_{0} .
\end{aligned}
$$

\section{Approximate Solutions}

In this section we first construct a two-parameter family of approximate solutions by using a similar method to [17] and then compute the error and last estimate the $H^{1} \times L^{2}$-norm of the error.

Following [17], our approximate solutions $u^{\omega, \lambda}=u^{\omega, \lambda}(t$, $x$ ) and $\rho^{\omega, \lambda}=\rho^{\omega, \lambda}(t, x)$ to (5) will consist of a low frequency and a high frequency part, that is,

$$
\begin{aligned}
u^{\omega, \lambda} & =\omega \lambda^{-1}+\lambda^{-s} \cos (\lambda x-\omega t), \\
\rho^{\omega, \lambda} & =\omega \lambda^{-1}+\lambda^{-s+1} \cos (\lambda x-\omega t),
\end{aligned}
$$

where $\omega$ is in a bounded set of $\mathbb{S}$ and $\lambda$ is in the set of positive integers $\mathbb{Z}_{+}$. 
Now we compute the error. Substituting the approximate solution $\left(u^{\omega, \lambda}, \rho^{\omega, \lambda}\right)$ into the first and second equation of (5), we get the following error:

$$
\begin{gathered}
E=u_{t}^{\omega, \lambda}+u^{\omega, \lambda} u_{x}^{\omega, \lambda} \\
+\partial_{x} \Lambda^{-2}\left(\left(u^{\omega, \lambda}\right)^{2}+\frac{1}{2}\left(u_{x}^{\omega, \lambda}\right)^{2}+\frac{1}{2}\left(\rho^{\omega, \lambda}\right)^{2}\right), \\
F=\rho_{t}^{\omega, \lambda}+u^{\omega, \lambda} \rho_{x}^{\omega, \lambda}+u_{x}^{\omega, \lambda} \rho^{\omega, \lambda} .
\end{gathered}
$$

Direct calculation shows that

$$
\begin{aligned}
& u_{t}^{\omega, \lambda}+ u^{\omega, \lambda} u_{x}^{\omega, \lambda} \\
&=-\frac{1}{2} \lambda^{-2 s+1} \sin (2 \lambda x-2 \omega t):=E_{1}, \\
& \partial_{x} \Lambda^{-2}\left(\left(u^{\omega, \lambda}\right)^{2}+\frac{1}{2}\left(u_{x}^{\omega, \lambda}\right)^{2}+\frac{1}{2}\left(\rho^{\omega, \lambda}\right)^{2}\right) \\
&=-\frac{3}{2} \lambda^{-2 s+1} \Lambda^{-2} \sin (2 \lambda x-2 \omega t) \\
& \quad-3 \omega \lambda^{-s} \Lambda^{-2} \sin (\lambda x-\omega t) \\
&+\frac{1}{2} \lambda^{-2 s+3} \Lambda^{-2} \sin (2 \lambda x-2 \omega t) \\
& \quad-\omega \lambda^{-s+1} \Lambda^{-2} \sin (\lambda x-\omega t) \\
&:= E_{2}+E_{3}+E_{4}+E_{5} .
\end{aligned}
$$

Similarly, we have

$$
\begin{aligned}
\rho_{t}^{\omega, \lambda} & +u^{\omega, \lambda} \rho_{x}^{\omega, \lambda}++u_{x}^{\omega, \lambda} \rho^{\omega, \lambda} \\
= & -\lambda^{-s} \sin (\lambda x-\omega t) \\
& -\lambda^{-2 s+2} \sin (2 \lambda x-2 \omega t) \\
:= & F_{1}+F_{2} .
\end{aligned}
$$

Let $C$ be a generic positive constant. For any positive quantities $P$ and $Q$, we write $P \lesssim Q(P \gtrsim Q)$ meaning that $P \leq C Q \quad(P \geq C Q)$ in the following.

Next, we estimate the error. We remark that the error of the periodic Camassa-Holm equation contains $E_{i}(i=1,2,3)$ and the estimate of $E_{i}$ was contained in paper [17]. In [17], they obtained that

$$
\begin{gathered}
\left\|E_{1}\right\|_{H^{1}},\left\|E_{2}\right\|_{H^{1}},\left\|E_{4}\right\|_{H^{1}} \lesssim \lambda^{-2 s+2}, \\
\left\|E_{3}\right\|_{H^{1}} \lesssim \lambda^{-s-1} .
\end{gathered}
$$

Now, we estimate $E_{5}$ and $F_{i}(i=1,2)$. We need the following lemma.

Lemma 5 (see [17]). Let $\sigma \in \mathbb{R}$. If $\lambda \in \mathbb{Z}_{+}$and $\lambda \gg 1$ then

$$
\|\cos (\lambda x-\alpha)\|_{H^{\sigma}(\mathbb{S})} \approx \lambda^{\sigma}, \quad \alpha \in \mathbb{R} .
$$

The above relation also holds if $\cos (\lambda x-\alpha)$ is replaced with $\sin (\lambda x-\alpha)$.
Estimating the $H^{1}$-Norm of $E_{5}$. By using the definition of $\Lambda$, we have

$$
\begin{aligned}
\left\|E_{5}\right\|_{H^{1}} & =\left\|\omega \lambda^{-s+1} \Lambda^{-2} \sin (\lambda x-\omega t)\right\|_{H^{1}} \\
& =\left|\omega \lambda^{-s+1}\right|\|\sin (\lambda x-\omega t)\|_{H^{-1}} \\
& \lesssim \lambda^{-s}
\end{aligned}
$$

where we used Lemma 5.

Estimating the $L^{2}$-Norms of $F_{1}$ and $F_{2}$. Also, we have

$$
\begin{gathered}
\left\|F_{1}\right\|_{L^{2}}=\left\|\lambda^{-s} \sin (\lambda x-\omega t)\right\|_{L^{2}} \lesssim \lambda^{-s} \\
\left\|F_{2}\right\|_{L^{2}}=\left\|\lambda^{-2 s+2} \sin (2 \lambda x-2 \omega t)\right\|_{L^{2}} \lesssim \lambda^{-2 s+2} .
\end{gathered}
$$

Collecting all error estimates together, we have the following Theorem.

Theorem 6. Let $s>5 / 2$. If $\omega$ is bounded, then for $\lambda \gg 1$ we have

$$
\|E\|_{H^{1}},\|F\|_{L^{2}} \lesssim \lambda^{-s}
$$

\section{Difference between Approximate and Actual Solutions}

In this section, we will estimate the difference between the approximate and actual solutions.

Let $\left(u_{\omega, \lambda}(t, x), \rho_{\omega, \lambda}(t, x)\right)$ be the solution to system (5) with initial data of the value of the approximate solution $\left(u^{\omega, \lambda}(t, x), \rho^{\omega, \lambda}(t, x)\right)$ at time zero; that is, $\left(u_{\omega, \lambda}(t, x)\right.$, $\left.\rho_{\omega, \lambda}(t, x)\right)$ satisfies

$$
\begin{gathered}
\partial_{t} u_{\omega, \lambda}+u_{\omega, \lambda} \partial_{x} u_{\omega, \lambda} \\
+\partial_{x} \Lambda^{-2}\left(u_{\omega, \lambda}^{2}+\frac{1}{2}\left(\partial_{x} u_{\omega, \lambda}\right)^{2}+\frac{1}{2} \rho_{\omega, \lambda}^{2}\right)=0 \\
t>0, x \in \mathbb{S} \\
\partial_{t} \rho_{\omega, \lambda}+u_{\omega, \lambda} \partial_{x} \rho_{\omega, \lambda}+u_{\omega, \lambda} \partial \rho_{\omega, \lambda}=0, \quad t>0, x \in \mathbb{S} \\
u_{\omega, \lambda}(0, x)=u^{\omega, \lambda}(0, x)=\omega \lambda^{-1}+\lambda^{-s} \cos (\lambda x), \quad x \in \mathbb{S}, \\
\rho_{\omega, \lambda}(0, x)=\rho^{\omega, \lambda}(0, x)=\omega \lambda^{-1}+\lambda^{-s+1} \cos (\lambda x), \quad x \in \mathbb{S} .
\end{gathered}
$$

Note that $\left(u_{\omega, \lambda}(0, x), \rho_{\omega, \lambda}(0, x)\right) \in H^{s} \times H^{s-1}, s \geq 0$. Moreover, we have

$$
\begin{gathered}
\left\|u_{\omega, \lambda}(0, x)\right\|_{H^{s}} \lesssim|\omega| \lambda^{-1}+1, \quad \lambda \gg 1, \\
\left\|\rho_{\omega, \lambda}(0, x)\right\|_{H^{s-1}} \lesssim|\omega| \lambda^{-1}+1, \quad \lambda \gg 1 .
\end{gathered}
$$

Therefore, if $s>5 / 2$, by using Theorems 1 and 4 , we have that, for any $\omega$ in a bounded set and $\lambda \gg 1$, problem (44) has a unique solution $z_{\omega, \lambda} \in C\left([0, T] ; H^{s}\right) \times C\left([0, T] ; H^{s-1}\right)$ with

$$
T \gtrsim \frac{1}{\left\|z_{\omega, \lambda}(0)\right\|_{H^{s} \times H^{s-1}}} \gtrsim \frac{1}{1+\lambda^{-1}} \gtrsim 1 .
$$


To estimate the difference between the approximate and actual solutions, we let

$$
v=u^{\omega, \lambda}-u_{\omega, \lambda}, \quad \sigma=\rho^{\omega, \lambda}-\rho_{\omega, \lambda} .
$$

Then $(\nu, \sigma)$ satisfies

$$
\begin{gathered}
v_{t}-v v_{x}+u^{\omega, \lambda} v_{x}+v u_{x}^{\omega, \lambda} \\
-\partial_{x} \Lambda^{-2}\left[v^{2}+\frac{1}{2} v_{x}^{2}+\frac{1}{2} \sigma^{2}-2 u^{\omega, \lambda} v\right. \\
\left.-u_{x}^{\omega, \lambda} v_{x}-\rho^{\omega, \lambda} \sigma\right]=E, \quad t>0, x \in \mathbb{S} \\
\sigma_{t}-v \sigma_{x}+u^{\omega, \lambda} \sigma_{x}+v \rho_{x}^{\omega, \lambda} \\
-\left(\sigma v_{x}-u^{\omega, \lambda} \sigma-\rho^{\omega, \lambda} v_{x}\right)=F, \quad t>0, x \in \mathbb{S} \\
v(0, x)=\sigma(0, x)=0, \quad x \in \mathbb{S}
\end{gathered}
$$

where $E$ and $F$ are defined as in Section 3.

Now we prove that the $H^{1}$-norm of difference decays.

Theorem 7. Let $s>5 / 2$; then

$$
\|v(t)\|_{H^{1}} \lesssim \lambda^{-s}, \quad\|\sigma(t)\|_{L^{2}} \lesssim \lambda^{-s}, \quad 0 \leq t \leq T, \lambda \gg 1 .
$$

Proof. Note that

$$
\begin{gathered}
\frac{1}{2} \frac{d}{d t}\|v(t)\|_{H^{1}}^{2}=\int_{\mathbb{S}}\left(v v_{t}+v_{x} v_{x t}\right) d x, \\
\frac{1}{2} \frac{d}{d t}\|\sigma(t)\|_{L^{2}}^{2}=\int_{\mathbb{S}} \sigma \sigma_{t} d x .
\end{gathered}
$$

Applying the operator $1-\partial_{x}^{2}=\Lambda^{2}$ to both sides of the first equations of system (48), we have

$$
\begin{aligned}
v_{t}= & \Lambda^{2} E-\Lambda^{2}\left(u^{\omega, \lambda} v_{x}-v u_{x}^{\omega, \lambda}\right) \\
& -\left(2 u^{\omega, \lambda} v+u_{x}^{\omega, \lambda} v_{x}+\rho^{\omega, \lambda} \sigma\right)_{x} \\
& +\frac{1}{2}\left(\sigma^{2}\right)_{x}+3 v v_{x}-2 v_{x} v_{x x}-v v_{x x x}+v_{x x t}, \\
\sigma_{t}=F- & \left(u^{\omega, \lambda} \sigma_{x}+v \rho_{x}^{\omega, \lambda}\right)-\left(u_{x}^{\omega, \lambda} \sigma+\rho^{\omega, \lambda} v_{x}\right)+(v \sigma)_{x} .
\end{aligned}
$$

Substituting (52) and (53) into (50) and (51), respectively, we obtain

$$
\begin{aligned}
\frac{1}{2} \frac{d}{d t}\|v(t)\|_{H^{1}}^{2}= & \int_{\mathbb{S}} v \Lambda^{2} \widetilde{E} d x-\int_{\mathbb{S}} v \Lambda^{2}\left(u^{\omega, \lambda} v_{x}+v u_{x}^{\omega, \lambda}\right) d x \\
& -\int_{\mathbb{S}} v\left(2 u^{\omega, \lambda} v+u_{x}^{\omega, \lambda} v_{x}+\rho^{\omega, \lambda} \sigma\right)_{x} d x
\end{aligned}
$$

$$
\begin{aligned}
& +\frac{1}{2} \int_{\mathbb{S}} v\left(\sigma^{2}\right)_{x} d x \\
& +\int_{\mathbb{S}}\left(v\left(3 v v_{x}-2 v_{x} v_{x x}-v v_{x x x}+v_{x x t}\right)\right. \\
& \left.+v_{x} v_{x t}\right) d x
\end{aligned}
$$

$$
\begin{aligned}
\frac{1}{2} \frac{d}{d t}\|\sigma(t)\|_{L^{2}}^{2}= & \int_{\mathbb{S}} \sigma \widetilde{F} d x-\int_{\mathbb{S}} \sigma\left(u^{\omega, \lambda} \sigma_{x}+v \rho_{x}^{\omega, \lambda}\right) d x \\
& -\int_{\mathbb{S}} \sigma\left(\rho^{\omega, \lambda} v_{x}+\sigma u_{x}^{\omega, \lambda}\right) d x+\int_{\mathbb{S}} \sigma(v \sigma)_{x} d x .
\end{aligned}
$$

It is direct to calculate that

$$
\begin{gathered}
\int_{\mathbb{S}}\left(v\left(3 v v_{x}-2 v_{x} v_{x x}-v v_{x x x}+v_{x x t}\right)+v_{x} v_{x t}\right) d x \\
=\int_{\mathbb{S}}\left[\left(v^{3}\right)_{x}-\left(v^{2} v_{x x}\right)_{x}+\left(v v_{x t}\right)_{x}\right] d x=0 .
\end{gathered}
$$

Substituting the above equalities into (54) and adding the resulting equations, we get

$$
\begin{aligned}
\frac{1}{2} \frac{d}{d t}( & \left.\|v(t)\|_{H^{1}}^{2}+\|\sigma(t)\|_{L^{2}}^{2}\right) \\
= & \int_{\mathbb{S}} v \Lambda^{2} \widetilde{E} d x+\int_{\mathbb{S}} \sigma \widetilde{F} d x \\
& -\int_{\mathbb{S}} v \Lambda^{2}\left(u^{\omega, \lambda} v_{x}+v u_{x}^{\omega, \lambda}\right) d x \\
& -\int_{\mathbb{S}} \sigma\left(u^{\omega, \lambda} \sigma_{x}+v \rho_{x}^{\omega, \lambda}\right) d x \\
& -\int_{\mathbb{S}} v\left(2 u^{\omega, \lambda} v+u_{x}^{\omega, \lambda} v_{x}+\rho^{\omega, \lambda} \sigma\right)_{x} d x \\
& -\int_{\mathbb{S}} \sigma\left(\rho^{\omega, \lambda} v_{x}+\sigma u_{x}^{\omega, \lambda}\right) d x \\
& +\int_{\mathbb{S}}\left[\frac{1}{2} v\left(\sigma^{2}\right)_{x}+\sigma(v \sigma)_{x}\right] d x \\
:= & I_{1}+I_{2}+\cdots+I_{7} .
\end{aligned}
$$

We first look at the last term $I_{7}$. Integrating by parts gives

$$
I_{7}=\int_{\mathbb{S}}\left[\frac{1}{2} v\left(\sigma^{2}\right)_{x}+\sigma(v \sigma)_{x}\right] d x=0 .
$$

Estimates of Integrals $I_{1}$ and $I_{2}$. Integrating by parts and applying the Cauchy-Schwarz inequality, we have

$$
\begin{gathered}
\left|\int_{\mathbb{S}} v \Lambda^{2} E d x\right|=\left|\int_{\mathbb{S}}\left(v E-v_{x} E_{x}\right) d x\right| \leq\|E\|_{H^{1}}\|v(t)\|_{H^{1}}, \\
\left|\int_{\mathbb{S}} \sigma F d x\right| \leq\|F\|_{L^{2}}\|\sigma(t)\|_{L^{2}} .
\end{gathered}
$$


Estimates of Integrals $I_{3}$. Integrating by parts, we get

$$
\begin{aligned}
-I_{3}= & \int_{\mathbb{S}} v \Lambda^{2}\left(u^{\omega, \lambda} v_{x}+v u_{x}^{\omega, \lambda}\right) d x \\
= & \int_{\mathbb{S}} v\left(u^{\omega, \lambda} v_{x}+v u_{x}^{\omega, \lambda}\right) d x \\
& +\int_{\mathbb{S}} v_{x}\left(u^{\omega, \lambda} v_{x}\right)_{x} d x+\int_{\mathbb{S}} v_{x}\left(v u_{x}^{\omega, \lambda}\right)_{x} d x
\end{aligned}
$$

and estimate its first part by

$$
\begin{aligned}
& \left|\int_{\mathbb{S}} v\left(u^{\omega, \lambda} v_{x}+v u_{x}^{\omega, \lambda}\right) d x\right| \\
& \quad \leq\left(\left\|u^{\omega, \lambda}(t)\right\|_{\infty}+\left\|u_{x}^{\omega, \lambda}(t)\right\|_{\infty}\right)\|v(t)\|_{H^{1}}^{2} .
\end{aligned}
$$

Its second part can be estimated by

$$
\begin{aligned}
\left|\int_{\mathbb{S}} v_{x}\left(u^{\omega, \lambda} v_{x}\right)_{x} d x\right| & =\left|\int_{\mathbb{S}}\left(u_{x}^{\omega, \lambda} v_{x}^{2}+\frac{1}{2} u^{\omega, \lambda}\left(v_{x}^{2}\right)_{x}\right) d x\right| \\
& =\frac{1}{2}\left|\int_{\mathbb{S}} u_{x}^{\omega, \lambda} v_{x}^{2} d x\right| \leq\left\|u_{x}^{\omega, \lambda}\right\|_{\infty}\|v(t)\|_{H^{1}}^{2}
\end{aligned}
$$

For the last part, integrating by parts, we obtain

$$
\begin{aligned}
\left|\int_{\mathbb{S}} v_{x}\left(v u_{x}^{\omega, \lambda}\right)_{x} d x\right| & \leq\left|\int_{\mathbb{S}} u_{x}^{\omega, \lambda} v_{x}^{2} d x\right|+\left|\int_{\mathbb{S}} v v_{x} u_{x x}^{\omega, \lambda} d x\right| \\
& \leq\left(\left\|u_{x}^{\omega, \lambda}(t)\right\|_{\infty}+\left\|u_{x x}^{\omega, \lambda}(t)\right\|_{\infty}\right)\|v(t)\|_{H^{1}}^{2}
\end{aligned}
$$

Estimates of Integrals $I_{4}$ and $I_{6}$. Integrating by parts, we can deal with the integral $I_{4}$ :

$$
\begin{aligned}
\left|I_{4}\right|=\left|-\int_{\mathbb{S}} \sigma\left(u^{\omega, \lambda} \sigma_{x}+v \rho_{x}^{\omega, \lambda}\right) d x\right| \\
=\left|\int_{\mathbb{S}} \frac{\sigma^{2}}{2} u_{x}^{\omega, \lambda}+v \sigma \rho_{x}^{\omega, \lambda} d x\right| \\
\leq\left(\left\|u_{x}^{\omega, \lambda}(t)\right\|_{L^{\infty}}+\left\|\rho_{x}^{\omega, \lambda}(t)\right\|_{L^{\infty}}\right) \\
\\
\times\left(\|\sigma\|_{L^{2}}^{2}+\|v(t)\|_{H^{1}}^{2}\right) .
\end{aligned}
$$

Similarly, we can estimate the term $I_{6}$,

$$
\begin{aligned}
\left|I_{6}\right|= & \left|-\int_{\mathbb{S}} \sigma\left(\rho^{\omega, \lambda} v_{x}+\sigma u_{x}^{\omega, \lambda}\right) d x\right| \\
\leq & \left(\left\|u_{x}^{\omega, \lambda}(t)\right\|_{L^{\infty}}+\left\|\rho^{\omega, \lambda}(t)\right\|_{L^{\infty}}\right) \\
& \times\left(\|\sigma\|_{L^{2}}^{2}+\|v(t)\|_{H^{1}}^{2}\right) .
\end{aligned}
$$

Estimate of the Integral $I_{5}$. Integrating by parts, we have

$$
\begin{aligned}
\left|I_{5}\right|= & \left|\int_{\mathbb{S}} v\left(2 u^{\omega, \lambda} v+u_{x}^{\omega, \lambda} v_{x}+\rho^{\omega, \lambda} \sigma\right)_{x} d x\right| \\
= & \left|\int_{\mathbb{S}} v_{x}\left(2 u^{\omega, \lambda} v+u_{x}^{\omega, \lambda} v_{x}+\rho^{\omega, \lambda} \sigma\right) d x\right| \\
\lesssim & \left(\left\|u^{\omega, \lambda}(t)\right\|_{\infty}+\left\|u_{x}^{\omega, \lambda}(t)\right\|_{\infty}+\left\|\rho^{\omega, \lambda}(t)\right\|_{\infty}\right) \\
& \times\left(\|v(t)\|_{H^{1}}^{2}+\|\sigma(t)\|_{L^{2}}^{2}\right) .
\end{aligned}
$$

Combining the estimations of $I_{1}-I_{7}$, we have that

$$
\begin{aligned}
& \frac{1}{2} \frac{d}{d t}\left(\|v(t)\|_{H^{1}}^{2}+\|\sigma(t)\|_{L^{2}}^{2}\right) \vdots\left(\|E\|_{H^{1}}+\|F\|_{H^{1}}\right)\left(\|v(t)\|_{H^{1}}+\|\sigma(t)\|_{L^{2}}\right) \\
&+\left(\left\|u^{\omega, \lambda}(t)\right\|_{\infty}+\left\|u_{x}^{\omega, \lambda}(t)\right\|_{\infty}+\left\|u_{x x}^{\omega, \lambda}(t)\right\|_{\infty}\right. \\
&\left.+\left\|\rho^{\omega, \lambda}(t)\right\|_{\infty}+\left\|\rho_{x}^{\omega, \lambda}(t)\right\|_{\infty}\right) \\
& \quad \times\left(\|v(t)\|_{H^{1}}^{2}+\|\sigma(t)\|_{H^{1}}^{2}\right) .
\end{aligned}
$$

Note that

$$
\begin{aligned}
& u^{\omega, \lambda}=\omega \lambda^{-1}+\lambda^{-s} \cos (\lambda x-\omega t), \\
& \rho^{\omega, \lambda}=\omega \lambda^{-1}+\lambda^{-s} \cos (\lambda x-\omega t) ;
\end{aligned}
$$

we have $\partial_{x} u^{\omega, \lambda}=-\lambda^{-s+1} \sin (\lambda x-\omega t)$. Furthermore, we have

$$
\begin{gathered}
\left\|u^{\omega, \lambda}\right\|_{L^{\infty}}, \lesssim \lambda^{-1}+\lambda^{-s}, \\
\left\|\partial_{x} u^{\omega, \lambda}\right\|_{L^{\infty}},\left\|\partial \rho^{\omega, \lambda}\right\|_{L^{\infty}} \lesssim \lambda^{-s+1}, \\
\left\|u_{x x}^{\omega, \lambda}\right\|_{L^{\infty}} \lesssim \lambda^{-s+2} .
\end{gathered}
$$

Submitting (69) into (67) and using Theorem 6, we obtain

$$
\begin{aligned}
& \frac{1}{2} \frac{d}{d t}\|z(t)\|_{H^{1} \times L^{2}}^{2} \\
& \quad \lesssim \lambda^{-s}\|z(t)\|_{H^{1} \times L^{2}}+\lambda^{-s+2}\|z(t)\|_{H^{1} \times L^{2}}^{2},
\end{aligned}
$$

which implies that

$$
\|z(t)\|_{H^{1} \times L^{2}} \lesssim \lambda^{-s}
$$

Noting that

$$
\|v(t)\|_{H^{1}},\|\varphi(t)\|_{L^{2}} \leq\|z(t)\|_{H^{1} \times L^{2}},
$$

combining the above inequality, we complete the proof of Theorem 7.

\section{Nonuniform Dependence}

In this section, we will prove nonuniform dependence for system (5) by taking advantage of the information provided by Theorems $1-4,6$, and 7 . Our main result is the following. 
Theorem 8. If $s>5 / 2$, then the data-to-solution $z(0) \rightarrow z(t)$ for system (5) is not uniformly continuous from any bounded subset of $H^{s} \times H^{s-1}$ into $C\left([-T, T] ; H^{s}\right) \times C\left([-T, T] ; H^{s-1}\right)$, where $z(0)=\left(u_{0}(x), \rho_{0}(x)\right)$ and $z(t)=(u(t, x), \rho(t, x))$. More precisely, there exist two sequences of solutions $\left(u_{\lambda}(t), \rho_{\lambda}(t)\right)$ and $\left(\widetilde{u}_{\lambda}(t), \tilde{\rho}_{\lambda}(t)\right)$ to the differential equations of (5) in $C\left([-T, T] ; H^{s}\right) \times C\left([-T, T] ; H^{s-1}\right)$ such that

$$
\begin{gathered}
\left\|u_{\lambda}(t)\right\|_{H^{s}}+\left\|\tilde{u}_{\lambda}(t)\right\|_{H^{s}}+\left\|\rho_{\lambda}(t)\right\|_{H^{s-1}}+\left\|\widetilde{\rho}_{\lambda}(t)\right\|_{H^{s-1}} \lesssim 1, \\
\lim _{\lambda \rightarrow \infty}\left\|u_{\lambda}(0)-\tilde{u}_{\lambda}(0)\right\|_{H^{s}}=\lim _{\lambda \rightarrow \infty}\left\|\rho_{\lambda}(0)-\widetilde{\rho}_{\lambda}(0)\right\|_{H^{s-1}}=0,
\end{gathered}
$$

$\liminf _{\lambda \rightarrow \infty}\left(\left\|u_{\lambda}(t)-\widetilde{u}_{\lambda}(t)\right\|_{H^{s}}+\left\|\rho_{\lambda}(t)-\widetilde{\rho}_{\lambda}(t)\right\|_{H^{s-1}}\right) \gtrsim \sin t$,

$$
|t|<T \leq 1
$$

Proof. Let $\left(u_{\lambda}(t), \rho_{\lambda}(t)\right)=\left(u_{1, \lambda}(t, x), \rho_{1, \lambda}(t, x)\right)$ and $\left(\tilde{u}_{\lambda}(t)\right.$, $\left.\tilde{\rho}_{\lambda}(t)\right)=\left(u_{-1, \lambda}(t, x), \rho_{-1, \lambda}(t, x)\right)$, where $\left(u_{1, \lambda}(t, x), \rho_{1, \lambda}(t, x)\right)$ and $\left(u_{-1, \lambda}(t, x), \rho_{-1, \lambda}(t, x)\right)$ are the unique solution to Cauchy problem (44) with initial data $\left(u^{1, \lambda}(0, x), \rho^{1, \lambda}(0, x)\right)$ and $\left(u^{-1, \lambda}(0, x), \rho^{-1, \lambda}(0, x)\right)$, respectively.

It follows from Theorem 1 that these solutions belong in $C\left([0, T] ; H^{s}\right) \times C\left([0, T] ; H^{s-1}\right)$. By (48) and the assumptions after Theorem 1 , we see that $T$ is independent of $\lambda \gg 1$. Letting $k=[s]+2$ and using estimate (34), we have

$$
\left\|u_{ \pm 1, \lambda}(t)\right\|_{H^{k}},\left\|\rho_{ \pm 1, \lambda}(t)\right\|_{H^{k-1}} \lesssim\left\|z^{ \pm 1, \lambda}(0)\right\|_{H^{k} \times H^{k-1}},
$$

where $z^{ \pm 1, \lambda}(0)=\left(u^{ \pm 1, \lambda}(0), \rho^{ \pm 1, \lambda}(0)\right)$ and $\left\|z^{ \pm 1, \lambda}(0)\right\|_{H^{k} \times H^{k-1}}^{2}=$ $\left\|u^{ \pm 1, \lambda}(0)\right\|_{H^{k}}^{2}+\left\|\rho^{ \pm 1, \lambda}(0)\right\|_{H^{k-1}}^{2}$. If $\lambda$ is large enough, then from Lemma 5 we have

$$
\begin{aligned}
\left\|u^{ \pm 1, \lambda}(t)\right\|_{H^{k}} \leq & \left\|u_{ \pm 1, \lambda}(t)\right\|_{H^{k}} \\
& +\lambda^{-(1 / 2) \delta-s}\left\|\phi\left(\frac{x}{\lambda^{\delta}}\right) \cos (\lambda x-\omega t)\right\|_{H^{k}} \\
\lesssim & \lambda^{-1+(1 / 2) \delta}+\lambda^{k-s}\|\phi\|_{2},
\end{aligned}
$$

which gives

$$
\left\|u^{ \pm 1, \lambda}(t)\right\|_{H^{k}} \lesssim \lambda^{k-s} .
$$

Combining (75) with (77), we obtain

$$
\left\|u_{ \pm 1, \lambda}(t)\right\|_{H^{k}} \lesssim \lambda^{k-s}, \quad \lambda \gg 1 .
$$

Estimate (77) together with (78) yields

$$
\left\|u^{ \pm 1, \lambda}(t)-u_{ \pm 1, \lambda}(t)\right\|_{H^{k}} \lesssim \lambda^{k-s}, \quad \lambda \gg 1 .
$$

Theorem 7 implies that

$$
\left\|u^{ \pm 1, \lambda}(t)-u_{ \pm 1, \lambda}(t)\right\|_{H^{1}} \lesssim \lambda^{-s}, \quad \lambda \gg 1 .
$$

Now, applying the interpolation inequality

$$
\|\varphi\|_{H^{s}} \leq\|\varphi\|_{H^{s_{1}}}^{\left(s_{2}-s\right) /\left(s_{2}-s_{1}\right)}\|\varphi\|_{H^{s_{2}}}^{\left(s-s_{1}\right) /\left(s_{2}-s_{1}\right)}
$$

with $s_{1}=1$ and $s_{2}=[s]+2=k$ and using estimates (79) and (80), we get

$$
\begin{aligned}
\left\|u^{ \pm 1, \lambda}(t)-u_{ \pm 1, \lambda}(t)\right\|_{H^{s}} \leq & \left\|u^{ \pm 1, \lambda}(t)-u_{ \pm 1, \lambda}(t)\right\|_{H^{1}}^{(k-s) /(k-1)} \\
& \times\left\|u^{ \pm 1, \lambda}(t)-u_{ \pm 1, \lambda}(t)\right\|_{H^{k}}^{(s-1) /(k-1)} \\
\leq & \lambda^{-s(k-s) /(k-1)} \lambda^{(k-s)(s-1) /(k-1)} \\
& \leqslant \lambda^{-(k-s) /(k-1)}, \quad \lambda \gg 1
\end{aligned}
$$

Hence

$$
\left\|u^{ \pm 1, \lambda}(t)-u_{ \pm 1, \lambda}(t)\right\|_{H^{s}} \lesssim \lambda^{-\varepsilon_{s}}, \quad \lambda \gg 1,
$$

where $\varepsilon_{s}=1 /(s+2)$.

Next, we prove (73) and (74). From (44), we have

$$
\begin{aligned}
\left\|u_{1, \lambda}(0)-u_{-1, \lambda}(0)\right\|_{H^{s}} & =\left\|2 \lambda^{-1}\right\|_{H^{s}} \\
& \approx \lambda^{-1} \longrightarrow 0 \text { as } \lambda \longrightarrow \infty, \\
\left\|\rho_{1, \lambda}(0)-\rho_{-1, \lambda}(0)\right\|_{H^{s-1}} & =\left\|2 \lambda^{-1}\right\|_{H^{s-1}} \\
& \approx \lambda^{-1} \longrightarrow 0 \text { as } \lambda \longrightarrow \infty,
\end{aligned}
$$

which implies that (73) holds.

Now, we prove (74). Obviously, we have

$$
\begin{aligned}
& \liminf _{\lambda \rightarrow \infty}\left(\left\|u_{\lambda}(t)-\widetilde{u}_{\lambda}(t)\right\|_{H^{s}}+\left\|\rho_{\lambda}(t)-\tilde{\rho}_{\lambda}(t)\right\|_{H^{s-1}}\right) \\
& \quad \geq \liminf _{\lambda \rightarrow \infty}\left\|u_{\lambda}(t)-\widetilde{u}_{\lambda}(t)\right\|_{H^{s}} .
\end{aligned}
$$

Thus we only prove that

$$
\liminf _{\lambda \rightarrow \infty}\left\|u_{\lambda}(t)-\widetilde{u}_{\lambda}(t)\right\|_{H^{s}} \gtrsim \sin t, \quad|t|<T \leq 1 .
$$

It is easy to see that

$$
\begin{aligned}
\left\|u_{1, \lambda}(t)-u_{-1, \lambda}(t)\right\|_{H^{s}} \geq & \left\|u^{1, \lambda}(t)-u^{-1, \lambda}(t)\right\|_{H^{s}} \\
& -\left\|u^{1, \lambda}(t)-u_{1, \lambda}(t)\right\|_{H^{s}} \\
& -\left\|u^{-1, \lambda}(t)-u_{-1, \lambda}(t)\right\|_{H^{s}} .
\end{aligned}
$$

It follows from (77) that

$$
\begin{array}{r}
\left\|u_{1, \lambda}(t)-u_{-1, \lambda}(t)\right\|_{H^{s}} \geq\left\|u^{1, \lambda}(t)-u^{-1, \lambda}(t)\right\|_{H^{s}}-c \lambda^{-\varepsilon_{s}}, \\
\lambda \gg 1,
\end{array}
$$

which implies that

$$
\begin{aligned}
& \liminf _{\lambda \rightarrow \infty}\left\|u_{1, \lambda}(t)-u_{-1, \lambda}(t)\right\|_{H^{s}} \\
& \quad \geq \liminf _{\lambda \rightarrow \infty}\left\|u^{1, \lambda}(t)-u^{-1, \lambda}(t)\right\|_{H^{s}} .
\end{aligned}
$$


Using the identity

$$
\cos \alpha-\cos \beta=-2 \sin \frac{\alpha+\beta}{2} \sin \frac{\alpha-\beta}{2}
$$

gives

$$
u^{1, \lambda}(t)-u^{-1, \lambda}(t)=2 \lambda^{-1}+2 \lambda^{-s} \sin (\lambda x) \sin t .
$$

Thus,

$$
\begin{aligned}
\left\|u^{1, \lambda}(t)-u^{-1, \lambda}(t)\right\|_{H^{s}} & \geq 2 \lambda^{-s}\|\sin \lambda x\|_{H^{s}}|\sin t|-2 \lambda^{-1}\|1\|_{H^{s}} \\
& \gtrsim \lambda^{-s}\|\sin \lambda x\|_{H^{s}}|\sin t|-\lambda^{-1}, \quad \lambda \gg 1 .
\end{aligned}
$$

Letting $\lambda \rightarrow \infty$ in the above inequality, we have

$$
\liminf _{\lambda \rightarrow \infty}\left\|u^{1, \lambda}(t)-u^{-1, \lambda}(t)\right\|_{H^{s}} \gtrsim|\sin t|
$$

Summing inequalities (89) and (93) up, it yields inequality (74). This completes the proof of this Theorem.

\section{Conflict of Interests}

The author declares that there is no conflict of interests regarding the publication of this paper.

\section{Acknowledgment}

The author was supported in part by PRC Grants NSFC 11226168 .

\section{References}

[1] A. Constantin and R. I. Ivanov, "On an integrable twocomponent Camassa-Holm shallow water system," Physics Letters A, vol. 372, no. 48, pp. 7129-7132, 2008.

[2] D. D. Holm, L. Náraigh, and C. Tronci, "Singular solutions of a modified two-component Camassa-Holm equation," Physical Review E, vol. 79, no. 1, Article ID 016601, 13 pages, 2009.

[3] J. Escher, O. Lechtenfeld, and Z. Yin, "Well-posedness and blowup phenomena for the 2-component Camassa-Holm equation," Discrete and Continuous Dynamical Systems, vol. 19, no. 3, pp. 493-513, 2007.

[4] Y. Fu and C. Qu, "Well posedness and blow-up solution for a new coupled Camassa-holm equations with peakons," Journal of Mathematical Physics, vol. 50, no. 1, Article ID 012906, 2009.

[5] C. Guan and Z. Yin, "Global existence and blow-up phenomena for an integrable two-component Camassa-Holm shallow water system," Journal of Differential Equations, vol. 248, no. 8, pp. 2003-2014, 2010.

[6] G. Gui and Y. Liu, "On the global existence and wave-breaking criteria for the two-component Camassa-Holm system," Journal of Functional Analysis, vol. 258, no. 12, pp. 4251-4278, 2010.

[7] G. Gui and Y. Liu, "On the Cauchy problem for the twocomponent Camassa-Holm system," Mathematische Zeitschrift, vol. 268, no. 1-2, pp. 45-66, 2011.

[8] R. Camassa and D. D. Holm, "An integrable shallow water equation with peaked solitons," Physical Review Letters, vol. 71, no. 11, pp. 1661-1664, 1993.
[9] A. Bressan and A. Constantin, "Global conservative solutions of the Camassa-Holm equation," Archive for Rational Mechanics and Analysis, vol. 183, no. 2, pp. 215-239, 2007.

[10] A. Constantin and J. Escher, "Well-posedness, global existence, and blowup phenomena for a periodic quasi-linear hyperbolic equation," Communications on Pure and Applied Mathematics, vol. 51, no. 5, pp. 475-504, 1998.

[11] Y. G. Fu and Z. R. Liu, "Non-uniform dependence on initial data for the periodic modified Camassa-Holm equation," Nonlinear Differential Equations and Applications, vol. 20, pp. 741-755, 2013.

[12] K. Grunert, H. Holden, and X. Raynaud, "Lipschitz metric for the periodic Camassa-Holm equation," Journal of Differential Equations, vol. 250, no. 3, pp. 1460-1492, 2011.

[13] H. Holden and X. Raynaud, "Global dissipative multipeakon solutions of the Camassa-Holm equation," Communications in Partial Differential Equations, vol. 33, no. 11, pp. 2040-2063, 2008.

[14] A. A. Himonas, G. Misiolek, and G. Ponce, "Non-uniform continuity in $\mathrm{H} 1$ of the solution map of the $\mathrm{CH}$ equation," Asian Journal of Mathematics, vol. 11, no. 1, pp. 141-150, 2007.

[15] A. A. Himonas, G. Misiołek, G. Ponce, and Y. Zhou, "Persistence properties and unique continuation of solutions of the Camassa-Holm equation," Communications in Mathematical Physics, vol. 271, no. 2, pp. 511-522, 2007.

[16] A. Himonas and C. Kenig, "Non-uniform dependence on initial data for the $\mathrm{CH}$ equation on the line," Differential Integral Equations, vol. 22, pp. 201-224, 2009.

[17] A. Himonas, C. Kenig, and M. Gerard, "Non-uniform dependence for the periodic CH equation," Communications in Partial Differential Equations, vol. 35, no. 6, pp. 1145-1162, 2010.

[18] H. Holden and X. Raynaud, "Global conservative solutions of the Camassa-Holm equation-a lagrangian point of view," Communications in Partial Differential Equations, vol. 32, no. 10, pp. 1511-1549, 2007.

[19] H. Holden and X. Raynaud, "Periodic conservative solutions of the Camassa-holm equation," Annales de l'Institut Fourier, vol. 58, no. 3, pp. 945-988, 2008.

[20] H. Holden and X. Raynaud, "Dissipative solutions for the camassa-holm equation," Discrete and Continuous Dynamical Systems, vol. 24, no. 4, pp. 1047-1112, 2009.

[21] Z. Yin, "Global existence for a new periodic integrable equation," Journal of Mathematical Analysis and Applications, vol. 283, no. 1, pp. 129-139, 2003.

[22] Y. Zhou and H. Chen, "Wave breaking and propagation speed for the CamassaHolm equation with $k \neq 0$," Nonlinear Analysis: Real World Applications, vol. 12, no. 3, pp. 1875-1882, 2011.

[23] J. Escher and Z. Yin, "Initial boundary value problems of the Camassa-Holm equation," Communications in Partial Differential Equations, vol. 33, no. 3, pp. 377-395, 2008.

[24] Q. Hu and Z. Yin, "Well-posedness and blow-up phenomena for a periodic two-component Camassa-Holm equation," Proceedings of the Royal Society of Edinburgh A, vol. 141, no. 1, pp. 93-107, 2011.

[25] G. Y. Lv, Y. H. P. Peter, and M. X. Wang, "Non-uniform dependence on initial data for the $\mu$-b equation," Zeitschrift für Angewandte Mathematik und Physik, vol. 64, pp. 1543-1554, 2013.

[26] G. Lv and M. Wang, "Non-uniform dependence for a modified Camassa-Holm system," Journal of Mathematical Physics, vol. 53, no. 1, Article ID 013101, 2012. 
[27] Q. Hu and Z. Yin, "Global existence and blow-up phenomena for a periodic 2-component Camassa-Holm equation," Monatshefte fur Mathematik, vol. 165, no. 2, pp. 217-235, 2012.

[28] R. Thompson, "The periodic cauchy problem for the 2component Camassa-Holm system," Differential Integral Equations, vol. 26, pp. 155-182, 2013.

[29] R. Iorio and I. de Magãlhaes, Fourier Analysis and Partial Differential Equation, Cambridge University Press, Cambridge, Mass, USA, 2001.

[30] M. Taylor, "Commutator estimates," Proceedings of the American Mathematical Society, vol. 131, pp. 1501-1507, 2003. 


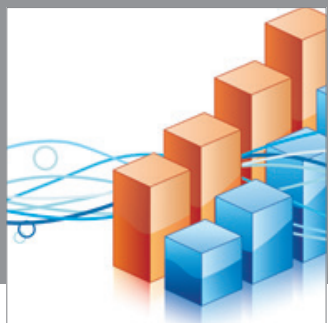

Advances in

Operations Research

mansans

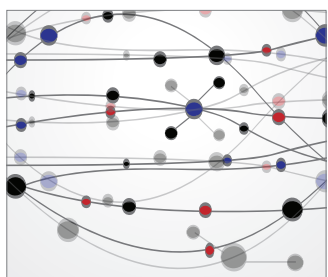

The Scientific World Journal
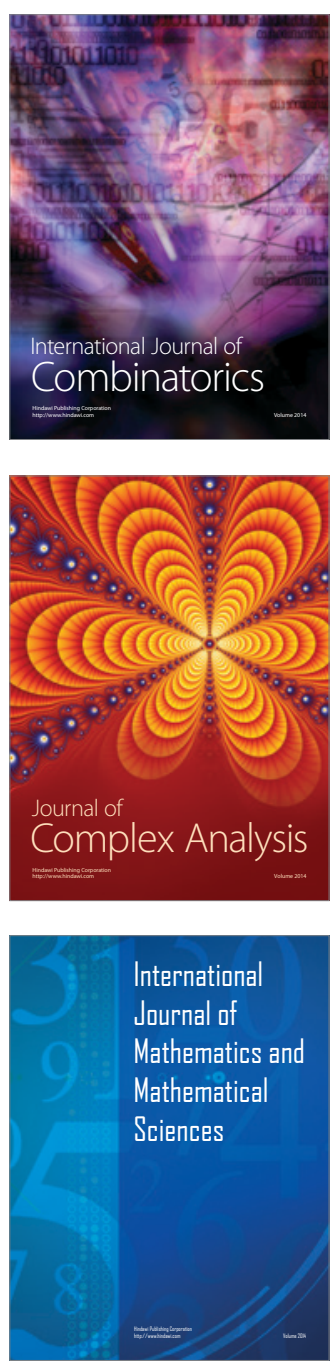
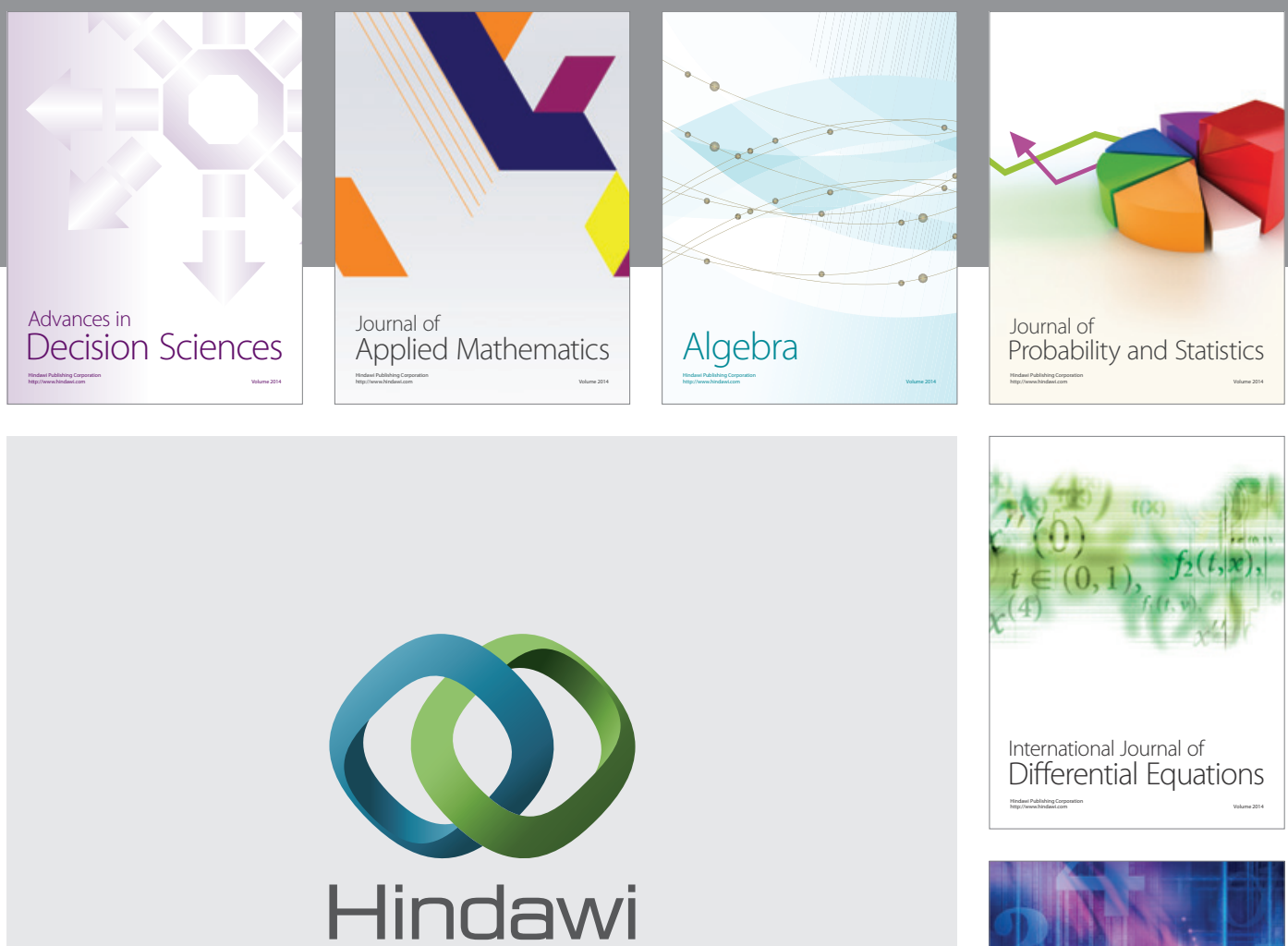

Submit your manuscripts at http://www.hindawi.com
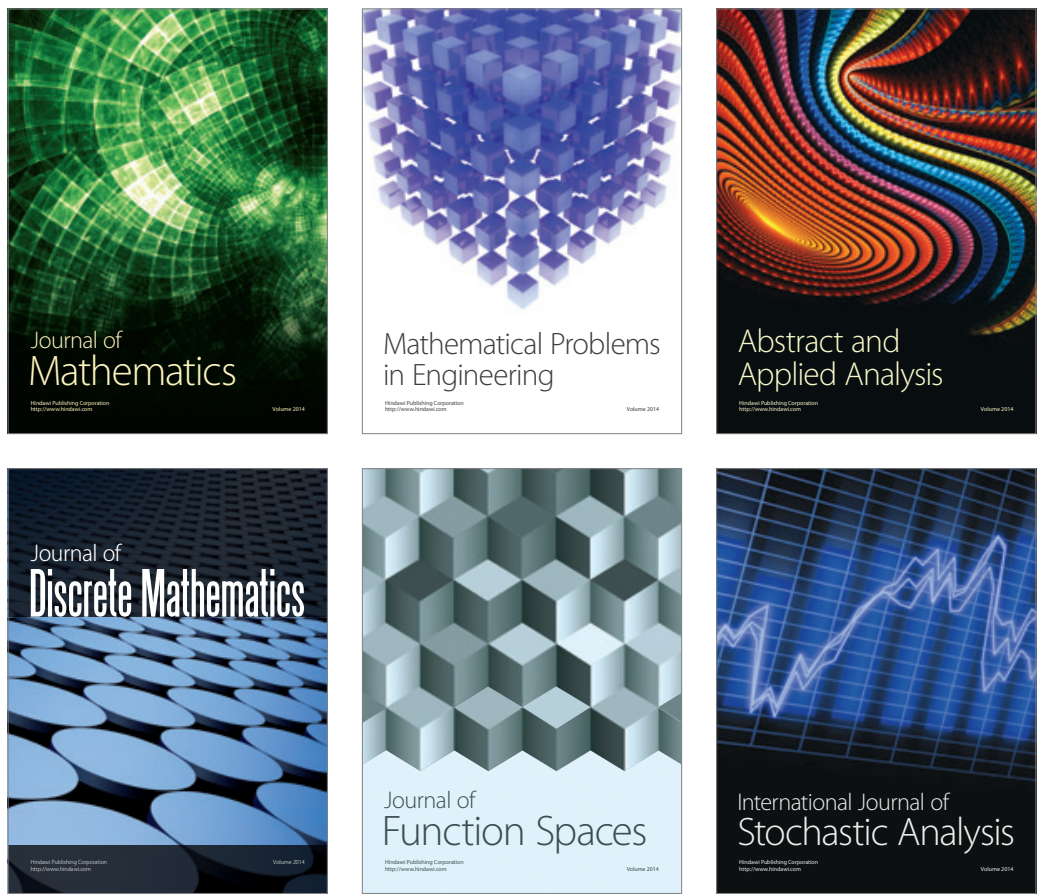

Journal of

Function Spaces

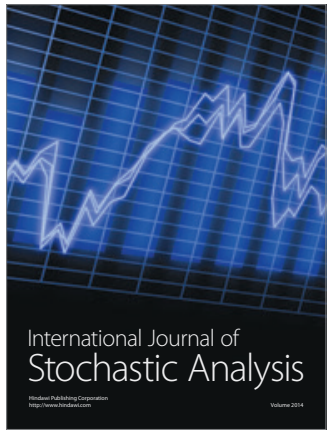

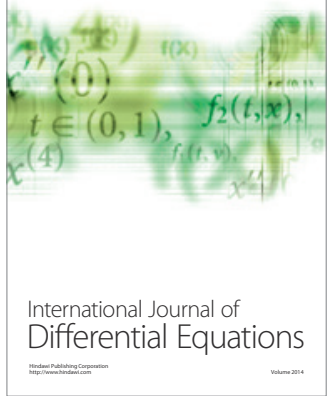
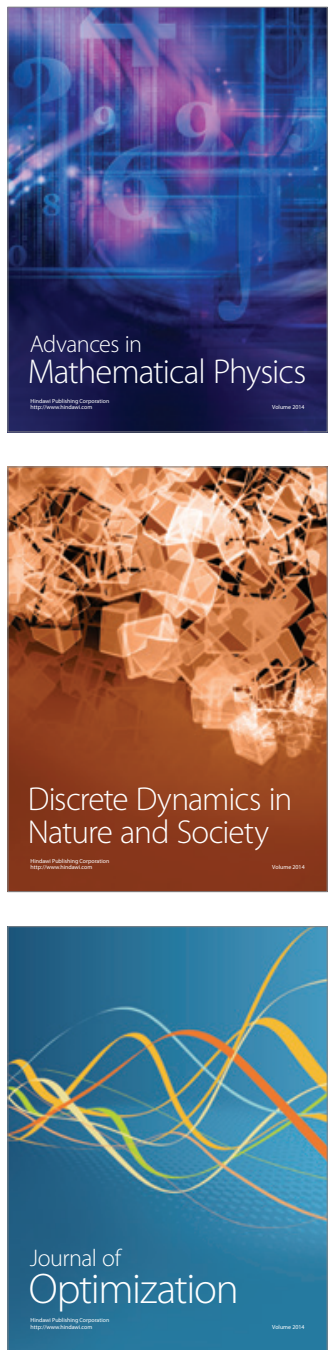